\title{
Special issue on exploring new Natural User Experiences
}

\author{
Francisco J. García-Peñalvo ${ }^{1}$ Lourdes Moreno ${ }^{2}$ (1)
}

Published online: 24 October 2017

(C) Springer-Verlag GmbH Germany 2017

The proliferation of new devices and interaction paradigm possibilities has enabled new forms of interaction between people and machines. Natural User Interfaces, which allow users to interact with a system using nothing but the human body, are one example of this. Users operate systems through intuitive actions such as gestures, touch, haptics or speech. This revolution, fostered by Natural User Interfaces, provides a great opportunity to enhance user experiences.

There is an increasing demand to create engaging and natural user experiences. These advancements allow for the design and development of more intuitive and engaging user interfaces that can provide universal access, support for assistive technologies for people with disabilities or rehabilitation applications.

This special issue comes after the successful organization of the 17th edition of the International Conference promoted by the Spanish Human-Computer Interaction Association (Spanish name: Asociación para la Interacción PersonaOrdenador, AIPO) [1]. This UAIS special issue is focused on research related with Natural User Experiences. As a result, eight papers describing new user interfaces, new methodologies and paradigms in the field of Natural User Interaction comprise this special issue.

Juan E. Garrido et al. present a new technique, the use of joint coordinates, to monitor patients in movement-based interaction systems in health care environments. This technique has been integrated into SIVIRE, a system developed

Lourdes Moreno

lmoreno@inf.uc3m.es

1 Computer Science Department, University of Salamanca, Salamanca, Spain

2 Computer Science Department, Universidad Carlos III de Madrid, Madrid, Spain by the authors, which provides a virtual online editor with the capacity to adapt rehabilitation exercises to patients' evolution. The authors present the results of their evaluation of the system implemented with this monitoring technique. The outcome proves that SIVIRE offers a reliable and useful tool for supporting rehabilitation therapies.

Roi Méndez et al. present a contribution in the virtual $\mathrm{TV}$ environment in which images from the real world are combined with virtual environments in order to obtain representations that give the impression of the real elements, such as actors or physical objects. In their paper, "Natural interaction in virtual TV sets through the synergistic operation of low cost sensors", a study analyzes the feasibility of the synergistic use of three different sensors (Optrictrack IR camera tracking system, Microsoft Kinect V2 and Leap Motion) to improve the interaction of the actors with the scene. It focuses mainly on natural gesture detection through the application of artificial intelligence techniques with the goal of using them during live broadcasts.

In previous works, Clara Bonillo et al. developed NIKVision, a vision-based tangible tabletop device designed for very young children and children with special needs. In their paper, "Tackling developmental delays with therapeutic activities based on tangible tabletops", they present the development of several activities with therapeutic goals for the tangible tabletop NIKVision geared towards children with developmental disorders. An evaluation was carried out with the children in order to verify the usefulness of these activities. Results indicate that thanks to this set of activities, it is possible to include tangible tabletop activities in therapies for these types of children so that they can benefit from the range of therapeutic possibilities offered by tangible interaction.

Alvaro Montero et al. present an approach for designing realistic and interactive Augmented Reality (AR) 
experiences in their paper, "Designing and Implementing Interactive and Realistic Augmented Reality Experiences". The approach combines the use of model-based AR techniques with the benefits that current game engines provide for the design and management of 3D virtual environments. Moreover, the authors carried out an evaluation assessing the effectiveness of the approach described in an event in which more than 100 people played AR games following the approach.

Manresa et al. present a natural user interface designed to provide access to mobile devices for motion-impaired people who cannot use the standard multi-touch input system to interact with tablets and smartphones. This natural user interface detects the user's head motion by means of a frontal camera. These different head positions are used to interact with the mobile device. An evaluation of the system's performance, when used by participants both with and without disabilities, is presented. The positive outcome indicates the potential of the interface for mobile accessibility for motionimpaired users who need alternative access to apparatuses in order to be able to interact with mobile devices.

In the paper by David Gonçalves et al. "Immersive $360^{\circ}$ video user experience-Impact of different variables in the sense of presence and cybersickness", different $360^{\circ}$ video formats combined with a variety of sound formats across genders are compared in order to understand their impact on the sense of presence and cybersickness felt in a virtual reality experience.

The number of on-board devices that provide information about vehicles, the driving process and the environment has increased. These devices can prove to be distracting for drivers. As an alternative to conventional HDD (Head-Down Display) screens, José A. Sánchez et al. present and evaluate a Head-Up Display Device (HUD) and speech recognition with fifty drivers, which may be good techniques to enrich the experience of drivers while maintaining high levels of safety. Results from the evaluation indicate that users agree that driving assistants combined with HUDs are useful and, at the same time, safe.

The paper "Towards the gestural interface: Comparative Analysis between Touch User Interfaces vs Gesture-based User Interfaces on Mobile Devices" by Juan A. HolgadoTerriza et al. evidences the competitive accuracy which Gesture-based User Interfaces (GBUI) can have in relation to classic touch user interfaces (TUI) as result of an evaluation carried out with twenty-five users in which a set of interaction tests were performed that compared two common anatomical positions (standing and lying down) individuals adopt when using smart phones.

In conclusion, as a whole, the papers included in this special issue are focused on research work concerned with the theory, practice and validation of the field of natural interaction in a variety of cases which can support universal access.

The guest editors would like to thank all the reviewers for their valuable input in addition to the authors who, at all times, carried out exemplary work in their research.

It is hoped that the readers will find the papers included in this special issue interesting.

\section{Reference}

1. Moreno López, L., Rubia Cuestas, E.J., Penichet, V.M.R., GarcíaPeñalvo, F.J. (eds.): Proceedings of the Interacción '16 Proceedings of the XVII International Conference on Human Computer Interaction (Salamanca, Spain-September 13-16, 2016). ACM, New York, NY, USA (2016) 